\title{
Nódulos linfáticos parietales del miembro pelviano en la inspección veterinaria de los ovinos
}

\author{
Polej, E.E. ; Fernández, J.A. ${ }^{2}$; Resoagli, J.M. ${ }^{2}$; F.Quintana, C.I. ${ }^{3}$ \\ ${ }^{1}$ Cátedra de Bromatología e Higiene Alimentaria, ${ }^{2}$ Cátedra de Anatomía, \\ ${ }^{3}$ Cátedra de Histología y Embriología, Fac. Cs. Veterinarias, UNNE, Cabral 2139, Corrientes, Argentina. \\ E-mail: carofloresq@gmail.com
}

\begin{abstract}
Resumen
Polej, E.E.; Fernández, J.A.; Resoagli, J.M.; F.Quintana, C.I.: Nódulos linfáticos parietales del miembro pelviano en la inspección veterinaria de los ovinos. Rev. Vet. 32: 1, 64-67, 2021. En la República Argentina la producción de carne ovina es impulsada por políticas del sector a fin de incrementar el volumen faenado y el consumo per cápita. Para certificar la inocuidad de estas carnes es indispensable la inspección sanitaria. En el post-mortem resulta imprescindible el examen de los nódulos linfáticos (NL) a fin de emitir un criterio sanitario sobre las reses inspeccionadas por parte del inspector veterinario en playa de faena frigorífica. Para ello, se identificó y describió forma, tamaño, situación, aferencias y eferencias de cada linfocentro. Para el estudio se utilizaron 8 ejemplares adultos de ambos sexos, a los cuales se les realizó eutanasia e inyección de tinta china a fin de que se visibilice el drenaje linfático para la ulterior disección. Se identificaron y describieron los linfocentros parietales externos del miembro pelviano, como ser los NL pre-femorales y NL poplíteos. Así mismo de igual manera se realizó con los linfocentros parietales internos del mismo miembro, estos fueron los NL retro-mamarios, NL inguinal superficial, NL isquiáticos, NL ilíacos externos, NL ilíacos internos y NL ilíacos medios. Los resultados obtenidos difieren en parte lo descrito por otros autores, debido principalmente a la inconstancia y a la no descripción de linfocentros por los mismos. No obstante, la descripción permitirá adecuar el sistema de inspección veterinaria post-mortem en playa de faena y de esta manera establecer los criterios sanitarios de reses faenadas e inspeccionadas.
\end{abstract}

Palabras clave: ovino, linfocentros, inspección, bromatología.

\begin{abstract}
Polej, E.E.; Fernández, J.A.; Resoagli, J.M.; F.Quintana, C.I.: Lymphatic parietal nodules of the bones pelvic member in the veterinary inspection of the sheep. Rev. Vet. 32: 1 , 64-67, 2021. In Republic of Argentina, ovine meat production is driven by sector politics in order to increase the slaughtered volume and the per capita consumption. Sanitary inspection is essential to certify the meat's innocuousness. In the post-mortem, the lymph nodes (LN) examination is essential in order to establish sanitary criteria on the cattle that have been inspected by the veterinary inspector in the slaughterhouse. For this purpose, the shape, size, location, afferences and efferences of each ly mphocenter were identified and described. For the study, 8 adult specimens of both sexes were used, which were euthanized and injected with chinese ink in order to visualize the lymphatic drainage for further dissection. The external parietal lymphocenters of the pelvic limb were identified and described, such as the pre-femoral and popliteal LN. The internal parietal lymphocenters of the same limb were also identified and described, such as retro mammary LN, superficial inguinal LN, ischial $\mathrm{LN}$, external iliac LN, internal iliac LN and middle iliac LN. The obtained results differ in part from those described by other authors; this is mainly due to the inconstancy and lack of description of lymphocenters by them. Nevertheless, the description will allow adapting the post-mortem veterinary inspection system in slaughter beach and in this way to establish the sanitary criteria of slaughtered and inspected cattle.
\end{abstract}

Key words: ovine, lymphocentres, inspection, bromatology 


\section{INTRODUCCIÓN}

La producción ovina representa un rubro significativo dentro del sistema agropecuario, siendo la cadena de carne y lana una de las más importantes del sector en Argentina ${ }^{9}$. En el año 2019 había en existencia a nivel nacional 14.774.196 cabezas, de las cuales el noreste argentino albergaba 2.089.589, representando el $14,2 \%$ del stock nacional. En ese mismo año se faenaron 918.999 cabezas, de las cuales se obtuvieron 13.785 toneladas de res con hueso, mientras que hasta agosto de 2020 se faenaron 617.920 cabezas, obteniendo 9.773 toneladas de res con hueso ${ }^{6}$.

El consumo de carne ovina per cápita en nuestro país es de $1,130 \mathrm{~kg} / \mathrm{ha}^{3}$. Para mejorar este indicador el entonces Ministerio de Agroindustrias presentó un programa de promoción, a los efectos de posicionar a la carne ovina como un alimento de ingesta habitual entre las demás carnes argentinas y fomentar su presencia en los mercados internos y externos ${ }^{5,7}$.

Para asegurar la inocuidad de las carnes es indispensable una inspección sistemática de la res, cabeza y vísceras, así como también la inspección de los linfocentros adquiere una relevancia significativa ya que entre otras funciones actúan como filtro ${ }^{1,4}$. Un linfocentro es un nódulo linfático o grupos de nódulos que aparecen constantemente en la misma región del cuerpo y reciben aferentes de esa región ${ }^{6}$.

La anatomía topográfica de los mismos es relativamente constante, manteniendo variaciones en números, tamaño, forma y ubicación entre las diversas especies de abasto. No obstante, es posible hallar variaciones dentro de una misma especie, pudiendo ser inconstantes algunos de estos linfocentros. Para ello resulta útil una descripción topográfica que facilite el abordaje para su inspección post-mortem en playa de faena.

Debido a ello el examen de este sistema reviste particular importancia para emitir un dictamen y determinar el destino de la res, siendo indispensable conocer la topografía y morfología de las linfoglándulas ${ }^{7}$.

Las estructuras linfáticas están ampliamente descriptas en la especie bovina, no así en el ovino, donde se las asocia con la topografía y morfología del bovino ${ }^{2,3,10}$, siendo el conocimiento de ello indispensable para la inspección post-mortem en playa de faena por parte del inspector veterinario.

La reglamentación sanitaria argentina (reglamento de inspección de productos, subproductos y derivados de origen animal; decreto 4238/68) no contiene especificaciones en cuanto a la inspección sanitaria obligatoria de ganglios linfáticos parietales y viscerales en la especie ovina ${ }^{8}$.

El presente trabajo tuvo como objetivo identificary describir la situación, forma y tamaño de los diferentes nódulos linfáticos (NL) de inspección en el cuarto posterior en playa de faena de la especie ovina, así como detallar los vasos aferentes y eferentes de cada uno de los linfocentros.

\section{MATERIAL Y MÉTODOS}

Se emplearon 8 ejemplares adultos de la especie ovina, machos y hembras, con un peso promedio de alrededor de $45 \mathrm{~kg}$. La eutanasia se realizó previa anestesia general con la combinación de maleato de acepromacina $(0,1 \mathrm{mg} / \mathrm{kg})$ y ketamina $(15 \mathrm{mg} / \mathrm{kg})$ vía endovenosa.

Posteriormente, se inyectó tinta china azul con aguja 25/8 y jeringa de $3 \mathrm{ml}$, en la piel de los espacios interdigitales de los miembros pelvianos, pared abdominal y zona perineal, a fin de que el drenaje linfático fuera visible en la disección.

Los especímenes se conservaron en cámara fría hasta su procesamiento. Para el estudio topográfico de los linfocentros, los NL se clasificaron en parietales o musculares externos e internos del miembro pelviano o cuarto posterior.

Los datos fueron registrados fotográficamente con cámara Nikon D 3200. Las mediciones se realizaron con calibre milimetrado y los valores numéricos fueron procesados estadísticamente para obtener media aritmética y desviación estándar.

\section{RESULTADOS Y DISCUSIÓN}

Una vez sacrificados, los ovinos fueron suspendidos con ganchos en el hueco del garrón para enseguida izarlos con aparejo. Luego se realizó el desollado y el eviscerado.

Parietales externos (NL prefemoral o precrural). Cuando está presente es único en cada cuarto, de forma elíptica, de 2,16 $\pm 0,52 \mathrm{~cm}$ de largo, $1,22 \pm 0,28 \mathrm{~cm}$ de ancho y $0,66 \pm 0,15 \mathrm{~cm}$ de espesor (Figura 1). Se halla situado en la aponeurosis de la cara craneal del músculo tensor de la fascia lata. Los aferentes provinieron de piel y tejido subcutáneo de la región abdominal, mientras que los eferentes drenaron en los vasos linfáticos ilíacos medios.

NL poplíteo: se halló profundamente en caudal y distal de la articulación genu, sobre el músculo gastronemio, medialmente de los músculos bíceps femoral y semitendinoso. De forma ovoide, aplanado lateralmente, rodeado de tejido adiposo, de $1,85 \pm 0,62 \mathrm{~cm}$ largo, $1,15 \pm 0,48 \mathrm{~cm}$ de ancho y $0,92 \pm 0,58 \mathrm{~cm}$ de espesor (Figura 2). Los vasos aferentes provinieron de la región distal del miembro; los vasos eferentes drenaron al nódulo linfático isquiático e iliofemoral

Parietales internos (NL retro mamarios en la hembra). Se situaron dorso caudalmente de la glándula mamaria. Se observaron 2-3 NL de forma circular, rodeados de tejido adiposo, con un diámetro de $1,2 \pm$ $0,47 \mathrm{~cm}$ (Figura 3). Los vasos aferentes provinieron de la glándula mamaria, piel y región femoral medial. Los vasos eferentes drenaron en los nódulos iliacos mediales o tronco lumbar.

NL inguinal superficial (en el macho). Se halla situado uno en cada cuarto, ubicados en caudal del anillo inguinal externo y de forma arriñonada: $1,8 \pm 0,357 \mathrm{~cm}$ 


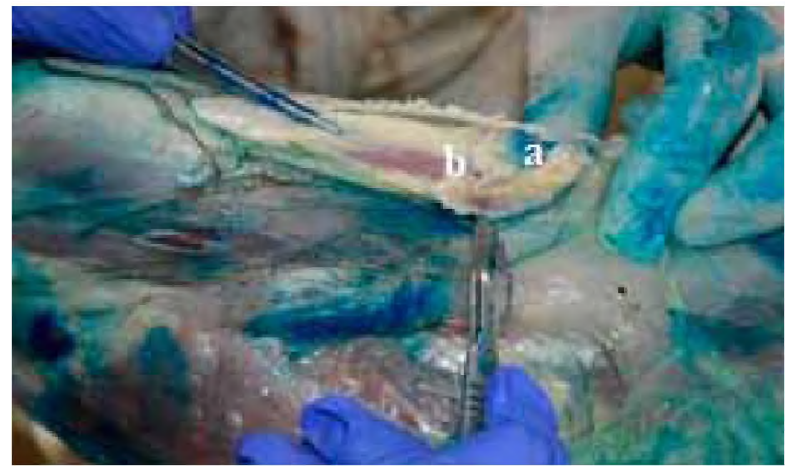

Figura 1. a-NL prefemoral, b-M tensor de la fascia lata.

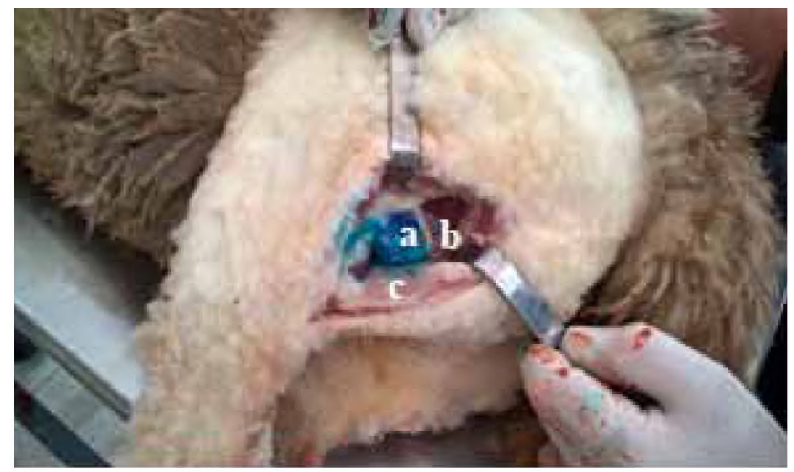

Figura 2. a-NL poplíteo, b-M gastronemio, c-M semitendinoso.

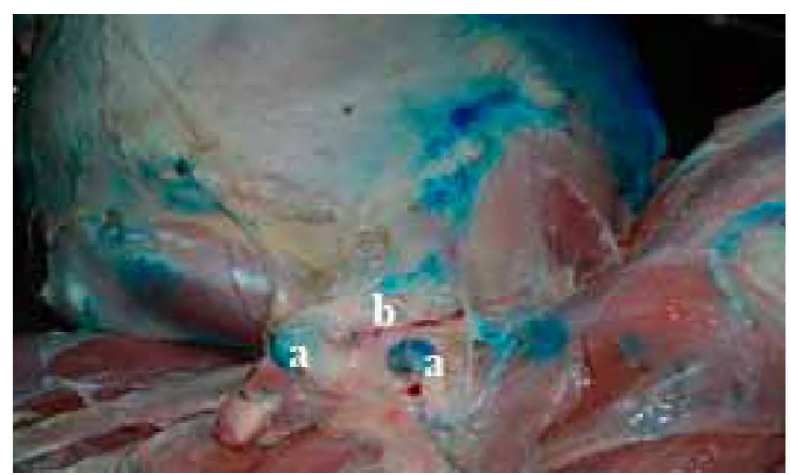

Figura 3. a-NL retromamario, b-G.mamaria.

de largo, $1 \pm 0,54 \mathrm{~cm}$ de ancho y $0,53 \pm 0,11 \mathrm{~cm}$ de espesor (Figura 4). Sus aferencias provinieron de los genitales externos y piel de la región. Sus eferencias drenaron en los NL ilíacos medios.

NL isquiáticos: se hallaron situados en la región pelviana, en ventral de los ligamentos sacroespinosos y tuberosos, a cada lado de la sínfisis púbica. De forma ovoide, de $1 \pm 0,3 \mathrm{~cm}$ de largo, $0,64 \pm 0,23 \mathrm{~cm}$ de ancho y $0,32 \pm 0,13 \mathrm{~cm}$ de espesor (Figura 5). Las aferencias provinieron de piel, músculos de la región glútea, cola y ano. Los vasos eferentes drenaron en los nódulos linfáticos ilíacos y lumbares.

NL ilíacos externos: el NL derecho estuvo situado en el inicio de la cuadrifurcación de la aorta y el NL izquierdo en ventral de la arteria iliaca externa y dorsal de la arteria ilíaca interna. De forma alargados y aplanados, con un tamaño de $1,6 \pm 1 \mathrm{~cm}$ de largo, $0,77 \pm$

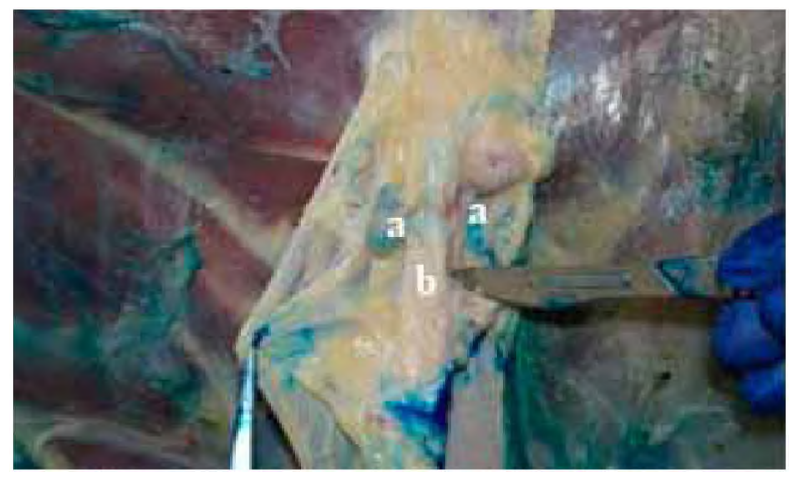

Figura 4. a-NL inguinal superficial, b-Región del anillo inguinal externo.

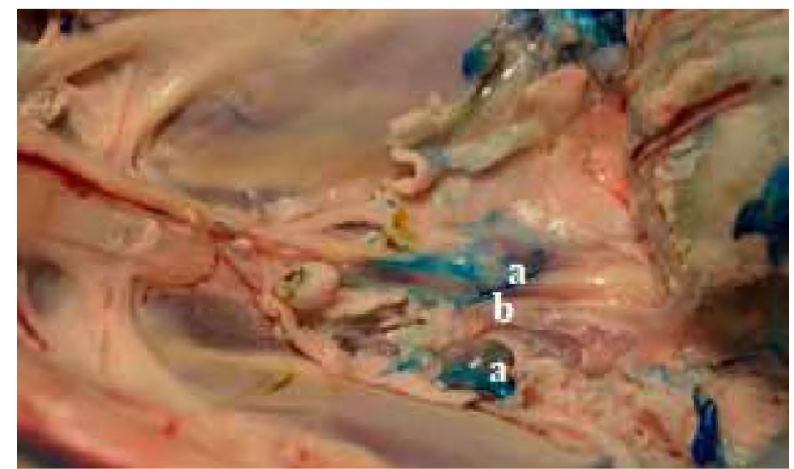

Figura 5. a-NL isquiático, b-sínfisis púbica

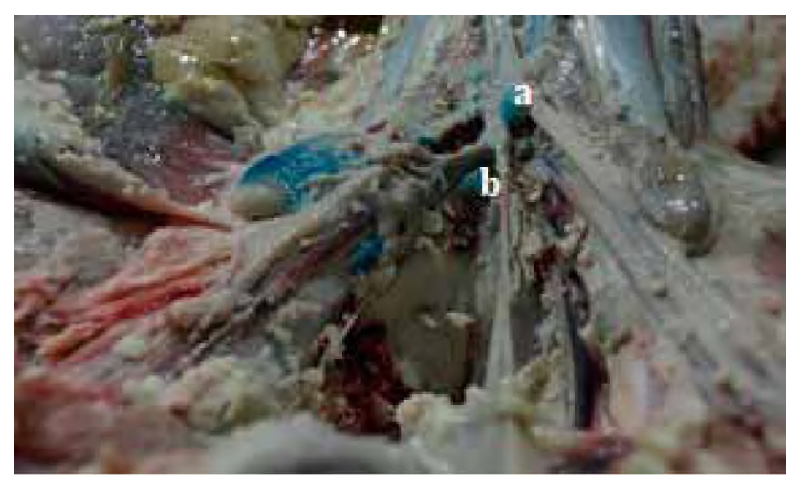

Figura 6. a-NL ilíaco externo derecho, b-NL ilíaco externo izquierdo

$0,37 \mathrm{~cm}$ de ancho y $0,3 \pm 0,1 \mathrm{~cm}$ de espesor (Figura 6). Sus vasos aferentes provinieron de genitales, abdomen, NL inguinales, precrurales e isquiáticos, y sus vasos eferentes drenaron en los NL sublumbares.

NL ilíacos internos: fueron inconstantes. Se halló el NL izquierdo en dos ejemplares, se situó en la cara medial del ligamento sacrotuberal, de forma ovoide y un tamaño de $0,85 \pm 0,63 \mathrm{~cm}$ de largo, $0,55 \pm 0,49 \mathrm{~cm}$ de ancho y $0,25 \pm 0,21 \mathrm{~cm}$ de espesor. Los vasos aferentes provinieron de la región inguinal y los eferentes drenaron los NL ilíacos medios.

NL ilíacos medios: se situaron próximos al origen de la arteria ilíaca circunfleja profunda e ilíaca interna y externa, de forma arriñonada y un tamaño $2,9 \pm 0,21$ $\mathrm{cm}$ de largo, $1,8 \pm 0,35 \mathrm{~cm}$ de ancho y $0,65 \pm 0,21 \mathrm{~cm}$ de espesor. Sus aferencias recibieron linfa de la pierna 
y región inguinal. Sus eferencias drenaron en el tronco lumbar.

Los NL descriptos en el ovino presentaron algunas variaciones con respecto a los que se describieron en el bovino. Tales diferencias fueron en tamaño y número, guardando una localización anatómica, aferencias y eferencias, un patrón similar al descrito en bovinos.

A diferencia de lo reseñado por varios autores $2,4,10$ se encontró inconstancia de los linfocentros precrurales en machos. Tal inconstancia se presentó en los ejemplares de manera unilateral o bilateral. En contraposición con los mismos autores que sostienen que el NL poplíteo es un ganglio único para cada cuarto, se halló en ejemplares NL poplíteos accesorios, con una ubicación anatómica contigua a los NL poplíteos.

No se describen en el ovino los NL ilíacos externos ${ }^{2}$, mientras que en los ejemplares analizados se localizó e identificó a los mismos en ambos cuartos del miembro pelviano, presentando el cuarto derecho un número de dos NL ilíacos externos.

En concordancia con autores ${ }^{2,3,10}$ hubo una inconstancia de los NL ilíacos internos, presentándose ésta en ambos sexos. No obstante, por las diferencias encontradas en este trabajo, al examen post-mortem en playa de faena la inspección es practicable mediante visualización, palpación e incisión, pudiendo de esta manera realizar un juicio valorativo fehaciente de la sanidad que presentan las reses faenadas e inspeccionadas, así como determinar su posterior destino.

\section{REFERENCIAS}

1. Bartels H. 1971. Inspección sanitaria de la carne, Edit. Acribia, Zaragoza, España.

2. Getty R. 1982. Anatomía de los animales domésticos, cap. 34: Sistema linfático de los rumiantes, Parte II, Ovinos, $5^{\circ}$ ed., Salvat, Barcelona, p. 1134-1176.

3. König H, Liebich H. 2005. Anatomía de los animales domésticos. II: Órganos, sistema circulatorio y sistema nervioso. Ed. Médica Panamericana, $2^{\mathrm{a}}$ ed., Buenos Aires, $400 \mathrm{p}$.

4. Mayer HF. 1984. Bromatología, higiene y control de alimentos, Ed. Impresiones UNNE, Resistencia (Chaco, Argentina), p. 57-59

5. Ministerio de Agricultura y Ganadería de la Nación. 1968. Reglamento de inspección de productos, subproductos y derivados de origen animal. Decreto 4238/68. Disponible en: www.alimentosargentinos.gov.ar

6. Ministerio de Agroindustrias. Informe mensual de carnes. Faena ovina. https:/www.magyp.gob.ar/sitio/areas/d_ ovinos/estadistica/existencias/index.php

7. Ministerio de Agroindustrias. Programa de promoción de carne ovina. https:/www. argentina.gob.ar/agroindustria.

8. Nómina Anatómica Veterinaria. 2012. Editorial Committee WAVA, 5th ed., Hannover (Germany), $166 \mathrm{p}$

9. Servicio Nacional de Sanidad y Calidad Agroalimentaria-SENASA. 2015. Ovinos. http://www.senasa.gob.ar/ cadena-animal/ovinos

10. Sisson S, Grossman JD. 1982. Anatomía de los Animales Domésticos, $5^{\circ}$ ed., Salvat, Barcelona. 